\title{
Apixaban in Patients with Thromboembolic Disease and Primary or Metastatic Brain Cancer?
}

\author{
Orwa Aboud* \\ Neuro Oncology Clinical Fellow, Skip Viragh Building, 9th Floor, Skip Viragh Building, 9th Floor, 201 North Broadway, Baltimore, MD 21287, USA
}

The Caravaggio trial [1] nicely documents that in patients with cancer and acute deep venous thrombosis or pulmonary emboli oral apixaban is as effective as subcutaneous dalteparin at preventing recurrent thromboembolic disease. In addition, anticoagulation with apixaban resulted in less "major" bleeding than dalteparin $(8.9 \%$ vs $11.4 \%)$. However, "clinically relevant non-major bleeding" occurred in 52 patients on apixaban versus 35 on dalteparin and "major bleeding or clinically relevant non-major bleeding" occurred in 70 patients on apixaban versus 56 on dalteparin. This study purposefully excluded patients with primary or metastatic brain tumors. These patient populations are important when it comes to anticoagulation. Thirtythree percent of all patients with solid tumors accrued to this study had cancer of the lung or breast. These two cancers account for over $60 \%$ of all patients who present with brain metastases [2]. In addition, over $30 \%$ of all patients with primary brain tumors develop thromboemboli requiring systemic anticoagulation [3]. Additional studies are required to document that apixaban can be safely administered to patients with primary and metastatic brain tumors.

\section{References}

1. Agnelli G, Becattini C, Meyer G (2020) Apixaban for the Treatment of Venous Thromboembolism Associated with Cancer. N Engl J Med 382:1599-1607.

2. Chamberlain MC, Baik CS, Gadi VK, Bhatia S, Chow LQ (2017) Systemic therapy of brain metastases: non-small cell lung cancer, breast cancer, and melanoma. Neuro Oncol 19: i1-i24. [Crossref]

3. Jo JT, Schiff D, Perry JR (2014) Thrombosis in brain tumors. Semin Thromb Hemost 40: 325-331. [Crossref]
Copyright: (C)2020 Aboud O. This is an open-access article distributed under the terms of the Creative Commons Attribution License, which permits unrestricted use, distribution, and reproduction in any medium, provided the original author and source are credited.
*Correspondence to: Orwa Aboud, M.D. Ph.D., Neuro Oncology Clinical Fellow, Skip Viragh Building, 9th Floor, Skip Viragh Building, 9th Floor, 201 North Broadway, Baltimore, MD 21287, USA, Tel: 410-614-9916; E-mail: oaboud1@jhmi.edu

Received: May 25, 2020; Accepted: June 03, 2020; Published: June 05, 2020 